\title{
The role of Primary Care Practitioner (PCP) in managing Inflammatory Bowel Disease (IBD) patients during COVID 19 Era
}

\author{
Fabio Ingravalle ${ }^{1}$, Giovanni Casella ${ }^{2}$, Adriana Ingravalle ${ }^{3}$, Claudio Monti $^{4}$, Enrico Santambrogio ${ }^{4}$, Fulvio Bonetti $^{4}$ and Aurelio Limonta $^{5}$ \\ ${ }^{1}$ School of Specialization in Hygiene and Preventive Medicine, University of Tor Vergata, Rome, 00133, Italy \\ ${ }^{2}$ General Practitioner, ATS Brianza, Limbiate (Monza), Italy \\ ${ }^{3}$ Department of Obstetrics and Gynecology, Campus Bio-Medico University, Rome, 00128, Italy \\ ${ }^{4}$ General Practitioner, ATS Brianza, Barlassina (Monza), Italy \\ ${ }^{5}$ General Practitioner, ATS Brianza, Concorezzo (Monza), Italy
}

\begin{abstract}
Introduction: The Covid-19 epidemic started in late 2019 from China. Since then, it has spread all over the world. SARS-CoV-2 infection poses a new challenge for the health systems of the countries involved, which have diverted all efforts to contain the pandemic spread, penalizing the management of patients with chronic diseases. Also, the activity of IBD units was limited and restricted to severe patients to avoid virus spreading.

Aim: The aim of the present review is to summarize and to report the earliest evidence regarding the novel managing strategies for IBD patients, in primary care.

Method: A review of the main database of scientific publications was conducted, regarding Covid-19 and the management of the patient with IBD in primary care.

Results: Several strategies have proven their efficacy. Among these, the most promising are telehealth and patient education ("shielding education"). These two interventions can monitor safely patient's health and reduce the infection risk in IBD patient.

Discussion: Nowadays, limited data in present scientific literature does not show that IBD patients have an increased risk of SARS-CoV-2 infection and a worst disease evolution than general population. The primary care practitioner can play a fundamental role in promoting the correct management of the IBD patient during the pandemic spread.
\end{abstract}

\section{What gap this fills}

\section{What is already known}

- Covid-19 is a threat for both patients with chronic diseases and primary care doctors.

- IBD patients require constant medical attention from both their primary care practitioner and their IBD unit.

\section{What this research add}

- Telehealth can reduce the risk of infection and at the same time maintain the same quality of care during Covid-19 pandemic.

- Primary care practitioner can play a fundamental role, because they can educate patients to avoid the risk of counting, as well as monitor their health situation.

\section{Introduction}

SARS-COV2 infection started from Hubei (China) [1] and in February 2020 spread in Italy. The first regions involved were Lombardy and Veneto, after that, in short time, all country areas. Suddenly a national lockdown was necessary [2]. Each state around the world has implemented its own pandemic response strategy. the main intervention carried out was a progressive reduction in the freedom of movement and aggregation of people. Access to hospitals was limited to covid-19 positive patients or critically ill patients by creating two different access routes [3]. So, the rapid diffusion of SARS-COV-2 had caused a critical condition in hospitals and in emergency departments. For this reason, National Healthcare systems were reorganized to contain virus spreading and improve patient's clinical management [45]. This healthcare reorganization has also affected Inflammatory Bowel Disease (IBD) Unit. The activity of IBD units was limited and restricted to severe patients to avoid virus spreading, but at the same time the correct quality standards of care was preserved for IBD patients [6-7].

A recent survey carried out in Lombardy, after 4 weeks since pandemic spread, find out that only the $31.3 \%$ of PCPs received a specific training on Covid-19 management, of which $67.1 \%$ was realized by online dedicated platforms. About $87.5 \%$ of the interviewed PCPs reported they have modified their day to day medical practice,

${ }^{\star}$ Correspondence to: Giovanni Casella, M.D., General Practitioner, ATS Brianza, Limbiate (Monza), 20812, Italy, Tel: 0039-347-3840325; E-mail caselgio@tiscali.it

Key words: SARS-COV-2, COVID-19, inflammatory bowel disease, telehealth, primary care

Received: October 19, 2020; Accepted: October 26, 2020; Published: October 29, 2020 
specifically using phone calls and telehealth systems. At least, most of interviewed PCPs adopted strategies to avoid waiting room overcrowding [8].

The implications of this situation stress, more than ever, the important role of Primary Care Practitioner (PCP) in helping IBD Unit and gastroenterologists to manage IBD patients. In fact, PCPs can be a fundamental link between the need for territorial patient management and access to specialist consulting, because PCPs are continuously improving their preparedness to Covid-19 emergency. This purpose could avoid a possible infection due to SARS-COV2.

\section{Data Sources and selection criteria}

We searched MEDLINE (PubMed), Web of Science and SCOPUS, from December 2019 to April 2020, in order to retrieve available data regarding to Covid-19 infection and IBD patient's management in primary care. A set of specific criteria were used for literature selection: Randomized controlled trials (RCT); prospective or retrospective cohort studies; reviews and meta analyses and international societies' guidelines. Only studies written in English with an available abstract were accepted.

\section{COVID 19 and IBD patients}

Patients with active IBD have a higher risk of infections than patients with a quiescent IBD. It was well proved this concerns both community and healthcare-associated infections, even in absence of immunosuppressive therapy [9]. However, the impact of immunosuppressive therapy during COVID-19 disease remains unclear [10]. Guan, et al. [1] reported data from an observational cohort of 1099 Chinese patients affected by Covid-19. The authors did not observe immunodeficiency as a risk factor for more severe disease. Disease severity was evaluated by radiological imaging and staged according to American Thoracic Society guidelines for Community Acquired Pneumonia [1]. Smoking, Sex Male and comorbidity as Arterial Hypertension, Vascular Disease, Diabetes may be considered as risk factors for a poor outcome in Covid-19 patients [11]. Surveillance Epidemiology of Coronavirus Under Research Exclusion (SECURE)-IBD Registry reported 239 (54\% male) cases of Covid-19 in IBD patients (137 with Crohn's Disease, 94 with Ulcerative Colitis, 5 with unclassified IBD) (9), of these 64 were hospitalized and 11 died [11]. In this report [12], among 11 died patients, only 5 were treated with Mesalazine, but they were 69 years old or older. 4 of these were on steroid therapy. British Society of Gastroenterology (BSG) has organized a specific IBD-Covid-19 Workshop Group in United Kingdom [10], where IBD patients were classified in 3 groups according Covid-19 risk: high, moderate and lowest (Table 1).

Another important aspect to consider is the psychological aspect that IBD patients fear of getting COVID-19. patients are aware that the drugs they take (such as steroids, azathioprine and biologics), can have an immune suppressive effect [13]. Bamias G, et al. [7] observed that the use of specific strategies, such as telemedicine services, electronic prescriptions and home delivery of medications, isolation of infusion units and IBD clinics in COVID-free zones of the hospitals could avoid interruption of appropriate therapies for IBD patients. For these reasons, the patient with IBD, as well as any patient suffering from chronicity, must not be left alone in this moment of pandemic emergency.

\section{The role of Primary Care Practitioner (PCP) in managing IBD patients during Covid-19 Era}

Covid-19 pandemic is a novel challenge for healthcare systems all over the world, so that a reorganization of the territorial health services is fundamental to find a solution and limit the spreading [10]. The primary medicine doctor can play a fundamental role in the organization of telemedicine because he knows both the patient and the area where he lives. In addition, clinics of primary medina physicians, properly sanitized according to ECDC guidelines, can receive chronic non-critical patients who may not have access to hospitals [14]. Of all the tools that technology makes available to the primary medicine physician, telemedicine is certainly the most advantageous, to prevent COVID-19 infection [15]. Moreover, Telehealth is cheap and easy to use, but unlucky its use is limited by licensing restrictions, healthcare private or public insurance, healthcare state funding, lack of appropriated infrastructures and the need to train operators for managing patients in telehealth [16]. Despite all, communications among patient, PCP and Specialists are still possible thanks to phone,

Table 1. IBD patient group classification according to IBD-Covid-19 Workshop Group

\begin{tabular}{|c|c|}
\hline IBD risk categories & Criteria \\
\hline Highest Risk "Shielding" & $\begin{array}{l}\text { - IBD patients with a Co-morbidity as Respiratory Disease, Cardiac Disease, Arterial Hypertension, Diabetes, aged } 70 \text { years or older, disease with } \\
\text { moderate-severe activity } \\
\text { - IBD patients of every age with assumption of }>20 \mathrm{mg} \text { Prednisolone/day, "Combined" therapy with Biologic and Immunosuppressive drugs or } \\
\text { - Systemic steroids within previous } 6 \text { weeks } \\
\text { - Short Gut Syndrome requiring Nutritional support } \\
\text { Requirement for parenteral nutrition }\end{array}$ \\
\hline $\begin{array}{l}\text { Moderate Risk "Stringent Social } \\
\text { Distancing" }\end{array}$ & $\begin{array}{l}\text { Patient on the following medications: } \\
\text { - Anti TNF (Infliximab, Adalimumab, Golimumab, Certolizumab) monotherapy, } \\
\text { - Biologic plus Immunomodulator in stable patient, } \\
\text { - Vedolizumab, } \\
\text { - } \text { Thtekinumab, } \\
\text { - Mepurine (Azathioprine, Mercaptopurine, Tioguanine), } \\
\text { - Calcineurin Inhibitors (Tacrolimus or Ciclosporin) } \\
\text { - Janus Kinase (JAK) Inhibitors (Tofacitinib), } \\
\text { - Mycophenolate Mofetil, Thalidomide, }\end{array}$ \\
\hline Lowest Risk "Social Distancing” & $\begin{array}{l}\text { Patients on following medications: } \\
\text { - } 5 \text {-ASA, } \\
\text { - Rectal therapies, } \\
\text { - } \text { Orally administered topically acting steroids (Budesonide or Beclomethasone), } \\
\text { - } \quad \text { Antidiarrheals (eg.Loperamide) } \\
\text { - Antibiotics for Bacterial Overgrowth or Perianal Disease }\end{array}$ \\
\hline
\end{tabular}


e-mail and dedicated helpline supported by a trained nurse. A senior review could act as "supervisor" for clinical and therapeutic decisions: this service may be considered as a "virtual safe clinic", but for work smoothly it is fundamental a good knowledge of patient's clinical history and patient's collaboration [10]. If all this is not possible, ECDC guidelines recommend that the patient be carefully informed about the procedures to be followed before, during and after the visit. Moreover, patient's anamnesis should be taken to rule out COVID-19-like respiratory symptoms or contacts with people with COVID-19. If the anamnesis was negative, the patient could arrange an appointment with the doctor in a COVID-free environment, otherwise the patient should carry out a test to investigate infection due to COVID-19 (14). ECCO and ACG guidelines recommend performing a test for COVID-19 in any doubtful situation [17-18].

The primary care physician, in addition to managing the basic therapy of IBD patients, can monitor the progress of therapy and can consult the gastroenterologist in case of relapse of the disease. Being the doctor of the patient and his family, he is able to monitor whether his patient is taking therapy correctly and can respond remotely or in person to the patient's needs without having to go to a hospital or other health facility [4-7].

Another fundamental role that the PCP can play is that of shielding education. As the GP can introduce the patient and his family to the correct personal and environmental hygiene procedures promoted by the national health system of his country. this strategy is widely applied in many countries and affects the entire general population, not just chronic patients $[2,5,14]$.

The rules that PCP must explain to IBD patients without Covid-19 Infection [10]:

1) Often wash your hands, as soon as, you get home. Avoid touching your face and keep 2 meters (6 ft) away from other people.

2) Work from home (if you can) and avoid contacts with sick people.

3) Stop Smoking (smoking increases the risk and severity of COVID-19).

4) Avoid non-essential travels, you should travel only for food, health reasons or work.

5) If it is possible, stay away from the hospital, if you need medical assistance contact your PCP or your IDB-unit.

6) Do not stop your medications without a medical instruction; medications prevent the disease "flare".

7) Ensure you have good supply of medications, because you must selfisolate to avoid Covid-19.

\section{The rules that PCP must explain to IBD patients with COVID Infection or with a household member affected [10]:}

1) Consider self-isolation and household quarantine.

2) Contact and inform IBD Team.

3) Consider to stop using medicines, only after IBD-Team indications, as Immunosuppressive (as Azathioprine, Methotrexate, Calcineurin Inhibitors, Mycophenolate, Thalidomide), Steroid (as Prednisolone), Biologic Drugs (as Infliximab, Adalimumab, Golimumab, Certolizumab; Vedolizumab; Ustekinumab) and small molecules (as Tofacitinib); the therapy may restart after an observational period of 14 days without symptoms;
4) Contact emergency staff as soon as clinical condition significantly deteriorates.

Proper informed patient may self-identify into a risk group and contact his GP and IBD-team [10]. This kind of interventions have already proved their efficacy. 318 Chinese IBD patients in Hubei had avoided infection thanks to early identification and intervention ("shielding" education) [19]. Clinical appointments by telephone or a formal telemedicine system are the best way to avoid direct contact among patients, that instead it would not be possible to avoid in a waiting room [7]. Fecal Calprotectin (FC) test may be limited by the presence of virus in the stools, but this test has several advantages. In fact, it is a potential alternative to endoscopy, and it is available as "point of care", feasible in every medical office, with results in few minutes [7]. FC testing with Partial Mayo/Simple Clinical Colitis Activity Index for Ulcerative Colitis and with Harvey-Bradshaw for Crohn's Disease can give crucial information in IBD patients managing without the possibility to perform an endoscopy.

IBD patients who have a medium-high risk class or patients with COVID-19, but without symptoms, should be monitored remotely, to verify that the symptomatic situation does not change. If the patient with IBD experiences symptoms of COVID-19 or a disease flare, the severity of both diseases should be assessed according to the scheme recommended by the guidelines [17-18].

\section{Discussion and Conclusions}

The limited data available [13] suggest that IBD patients do not seem to have an increased risk of infection with SARS-CoV-2 or an increased severity of development of COVID-19 [14]. The inflammation of the bowel may be considered as a risk for SARS-CoV-2 infection, but further studies are needed to confirm and quantified this risk. The goal of PCP and IBD team is to maintain a clinical remission of the disease and to reduce the risk of "flare". This achieve avoid the need of more intense medical therapy or hospitalization [13]. The recommendations for IBD population are like those for general population such as strict social distancing, work from home, a meticulous hands hygiene and avoiding contacts with infected individuals [13]. PCP have always the fundamental role in protecting and assure day to day care to vulnerable patients. The Covid-19 pandemic represents a novel challenge, which can be overcome thanks to telehealth, virtual counselling between the patient and the doctor, drug home delivery and many other strategies which are becoming become a new way to implement health strategies for the future.

\section{Acknowledgement}

No Ethical Committee Approval is required for the present review.

\section{Funding}

None.

\section{Conflict of interest}

None.

\section{References}

1. Guan W-Jie, Ni Z-yi, Hu Y, Liang WH, Ou Cq, et al. (2020) Clinical characteristics of Coronavirus disease 2019 in China. New England J Med 382:1708-1720.

2. http://www.protezionecivile.gov.it/

3. Loeffler-Wirth H, Schmidt M, Binder H (2020) Covid-19 Transmission TrajectoriesMonitoring the Pandemic in the Worldwide Context. Viruses 12: 777. [Crossref] 
4. Danese S, Cecconi M, Spinelli A (2020) Management of IBD during the COVID-19 outbreak: resetting clinical priorities. Nat Rev Gastroenterol Hepatol [Crossref]

5. Zongo P, Zorom M, Mophou G, Dorville R, Beaumont C (2020) A model of COVID-19 transmission to understand the effectiveness of the containment measures: application to data from France. Epidemiol Infect 148: e221. [Crossref]

6. Allocca M, Fiorino G, Furfaro F, Gilardi D, Radice S, et al. (2020) Maintaining the quality standards of care for Inflammatory Bowel Disease Patients during the COVID-19 pandemic. Clin Gastroenterol Hepatol 8:1882-1883 [Crossref]

7. Bamias G, Lagou S, Gizis M, Karampekos G, Kyriakoulis KG, et al. (2020) The Greek Response to COVID-19: A True Success Story from an IBD Perspective. Inflamm Bowel Dis 26: 1144-1148. [Crossref]

8. Fiorino G, Colombo M, Natale $\mathrm{C}$ et al. Clinician Education and Adoption of Preventive Measures for COVID-19: A Survey of a Convenience Sample of General Practitioners in Lombardy, Italy. Ann Intern Med 15: M20-1447. [Crossref]

9. Lichtenstein GR, Feagan BG, Cohen RD (2012) Serious infection and mortality in patients with Crohn's Disease: more than 5 years of follow-up in the "TREAT" registry. Am J Gastroenterol 107: 1409-1422 [Crossref]

10. Kennedy NA, Jones GR, Lamb CA, Appleby R, Arnott I, et al. (2020) British Society of Gastroenterology guidance for management of Inflammatory Bowel Disease during the COVID-19 pandemic. Gut 69: 984-990. [Crossref]

11. Wenham C, Smith J, Morgan R (2020) OVID-19: the gendered impacts of the outbreak Lancet 395: 846-848 [Crossref]
12. https://covidibd.org/current-data/

13. Ciacci C, Siniscalchi M (2020) Tips from the battlefront: Psychological support of patients with a chronic illness during the COVID-19 lockdown in four steps. United European Gastroenterol $J$ 8: 741-742. [Crossref]

14. European Centre for Disease Prevention and Control (2020) COVID-19 infection prevention and control for primary care, including general practitioner practices, dental clinics and pharmacy settings - 9 June 2020. ECDC: Stockholm.

15. Smith AC, Thomas E, Snoswell CL, Haydon H, Mehrotra A, et al. (2020) Telehealth for global emergencies: Implications for Coronavirus Disease 2019 (COVID-19). $J$ Telemed Telecare 26:309-313 [Crossref]

16. Hoolander JE, Carr BG (2020) Virtually Perfect? Telemedicine for COVID-19. N Engl J Med 382: 1679-1681 [Crossref]

17. Rubin TD, Feuerstein JD, Wang AY, Cohen RD, et al. (2020) AGA Clinical Practice Update on Management of Inflammatory Bowel Disease during the COVID-19 Pandemic: Expert Commentary. Gastroenterology 159: 350-357. [Crossref]

18. ECCO update on COVID-19 and IBD (2020) Update on COVID19 for patients with Crohn's Disease and Ulcerative Colitis. IOIBD.

19. An P, Ji M, Ren H, Su J, Ding NS, et al. Protection of 318 Inflammatory Bowel Disease Patients from the Outbreak and Rapid Spread of COVID-19 Infection in Wuhan, China. Lancet Gastroenterol Hepatol 5: 525-527. [Crossref]

Copyright: (C2020 Ingravalle F. This is an open-access article distributed under the terms of the Creative Commons Attribution License, which permits unrestricted use, distribution, and reproduction in any medium, provided the original author and source are credited. 